\title{
Cultura gerencial y cambio organizacional en Cierres Ideal de México: un "modelo" de calidad guadalupano
}

\author{
Gabriela García Figueroa y Sergio A. Sandoval Godoy
}

Este documento aborda los procesos de racionalidad y cultura gerencial asociados a la implementación de un nuevo modelo de organización del trabajo en la empresa Cierres Ideal de México. Se enfatiza el discurso y las formas de dirección de la gerencia, así como el manejo de elementos subjetivos de influencia, como valores, incentivos y culto religioso. De igual forma, da cuenta de las contradicciones, inercias y particularidades de esta empresa en la aventura del cambio organizacional. Así, se introduce en el espacio de las significaciones y las identificaciones en el trabajo y en cómo se asumen, aceptan o rechazan las nuevas reglas y principios que hay detrás de los programas de calidad total que se supone se desarrollan en la empresa estudiada.

PALABRAS CLAVE: cultura, racionalidad, calidad, influencia y organización del trabajo

\section{Management Culture and Organizational Change in Cierres Ideal of Mexico: a Guadalupano Quality "Model”}

This paper addresses the processes of rationality and managerial culture related to the implementation of a new model of work organization in the Mexican company Cierres Ideal. It emphasizes not only the speech and forms of management administration, but also the management of influential subjective elements such as values, incentives and worship. Similarly, the contradictions, inertia and particularities of this company in the adventure of organizational change are described. Thus, the article explores the space of meanings and identities at work and how the new rules and principles that are behind of the total quality programs -that are supposed to be developed in the studied company-, are assumed, accepted or rejected.

KEYWORDS: culture, rationality, quality, influence and work organization

GABRIELA GARCía FiguerOA: El Colegio de Sonora, Hermosillo, Sonora ggarcia@colson.edu.mx

SERgio A. SANdoval GodoY: Centro de Investigación en Alimentación y Desarrollo, A. C., Hermosillo, Sonora ssandoval@ciad.mx 


\section{INTRODUCCIÓN}

$\mathrm{D}$ esde inicios de la década de 1980, el discurso de la racionalidad económica basada en la ganancia y la reducción de costos de producción ha sido paulatinamente sustituido por el de una racionalidad económica basada en la calidad de los productos, el desarrollo del personal y la humanización del trabajo. La revolución tecnológica y las transformaciones asociadas a las nuevas formas organizacionales han planteado a las gerencias la instrumentación de nuevas estrategias productivas y culturales que posibiliten la coexistencia de intereses comunes a trabajadores y empresarios. En gran parte, la base de dichas estrategias ha girado en torno al desarrollo de los llamados "sistemas sociotécnicos", cuyo componente central son los "equipos autónomos de trabajo" y los "círculos de calidad", a los que teóricamente se les delega la responsabilidad de dirección, ejecución y organización de los sistemas productivos.

Las expresiones de estos cambios han sido de naturaleza diversa, y sus resultados, hasta hoy, ampliamente discutidos. Para los estudiosos del tema, la incógnita de partida es si efectivamente existe en las plantas manufactureras una nueva racionalidad basada en la humanización del trabajo, o si sólo se trata de un nuevo discurso gerencial que busca adaptar el objetivo de la ganancia capitalista a los modernos cambios socioproductivos. Los desacuerdos no parecen ser significativos cuando se refieren a dicho objetivo; sin embargo, las diferencias de fondo giran alrededor de tres maneras distintas de abordar el problema: la primera, una posición fundamentalista que plantea que los medios y fines que persiguen las empresas son inamovibles y aplicables a todas las situaciones; la segunda, una posición contingencialista que señala que éstos van orientados a la adaptación del entorno; y la tercera, una posición procesualista que sugiere que la combinación de medios y fines puede desarrollarse en varias direcciones dependiendo de la historia y de la trayectoria propia de la empresa ${ }^{1}$.

\footnotetext{
${ }^{1} \mathrm{Al}$ respecto, véase Thevenet, 1992. Según este autor, para descubrir la lógica inherente a las gerencias es importante acudir a claves culturales que revelan el fundamento de las prácticas de dirección, su cohe-
}

En el caso Cierres Ideal de México (CIMSA), empresa ubicada en el Valle de México y objeto de estudio de este trabajo, los problemas generados por la introducción de nuevas formas organizacionales han sido sobre todo de adaptación al entorno y de integración de los recursos humanos. A pesar de que la gerencia promueve la aplicación de un modelo ideal de organización del trabajo, en la práctica es menos partidaria de buscar soluciones universales a los problemas en la línea de producción y más afín a adaptarse a las situaciones cambiantes del entorno. Su aplicación ha rebasado ya el ámbito de lo económico, porque su esencia se inscribe ahora en los espacios de la cultura y la subjetividad, de los valores compartidos o colectivos que le dan sentido a su acción y a los resultados de la misma. Así, trataremos de probar que la racionalidad y la cultura gerencial ${ }^{2}$ asociada a un nuevo modelo organizacional en dicha empresa deben ser vistas como un fenómeno contradictorio y engañoso, debido a: 1) una ruptura entre los planteamientos del modelo y las expectativas de comunicación y liderazgo compartido creadas en los trabajadores, lo que sugiere un control de calidad discrecional sui generis; 2) una combinación de elementos de formas tradicionales de organización burocrática con formas participativas de trabajo en equipo, y 3) una manipulación de valores y prácticas

rencia interna y, sobre todo, las eventuales paradojas y oportunidades de cambio. En este sentido critica las concepciones estereotipadas y universales, así como las contingentes y funcionalistas, y se pronuncia a favor de los análisis de la cultura que valora el proceso de desarrollo empresarial a lo largo de una historia en la que el tiempo permite el aprendizaje de formas apropiadas de acción y resolución de problemas.

${ }^{2}$ Entendemos por cultura gerencial un ámbito constituido no homogéneamente donde se articulan procesos de creación de sentido u ordenación del sentido de la realidad gerencial, a través de prácticas sociales e institucionales. La idea es que esta no coherencia es la que deja paso a la posibilidad de recambios y reconfiguraciones subjetivas en la cultura gerencial, lo que nos sitúa frente a la posibilidad de una subjetividad dinámica en la que los individuos crean sus propias subjetividades o sentidos para la acción de acuerdo con situaciones determinadas. Cultura gerencial como ámbito de significaciones de lo que es dirigir u ordenar; del sentido de las formas autoritarias o por convencimiento en que se implementan los cambios en materia de organización del trabajo a los subordinados; significación de las doctrinas gerenciales y de su aplicación práctica, de la ganancia y el prestigio, de competir, de su pertenencia a un grupo social y a una estructura jerárquica dentro de una empresa, del respeto y la autoridad, de controlar, de influir, del poder; significación de lo que es ser gerente. 


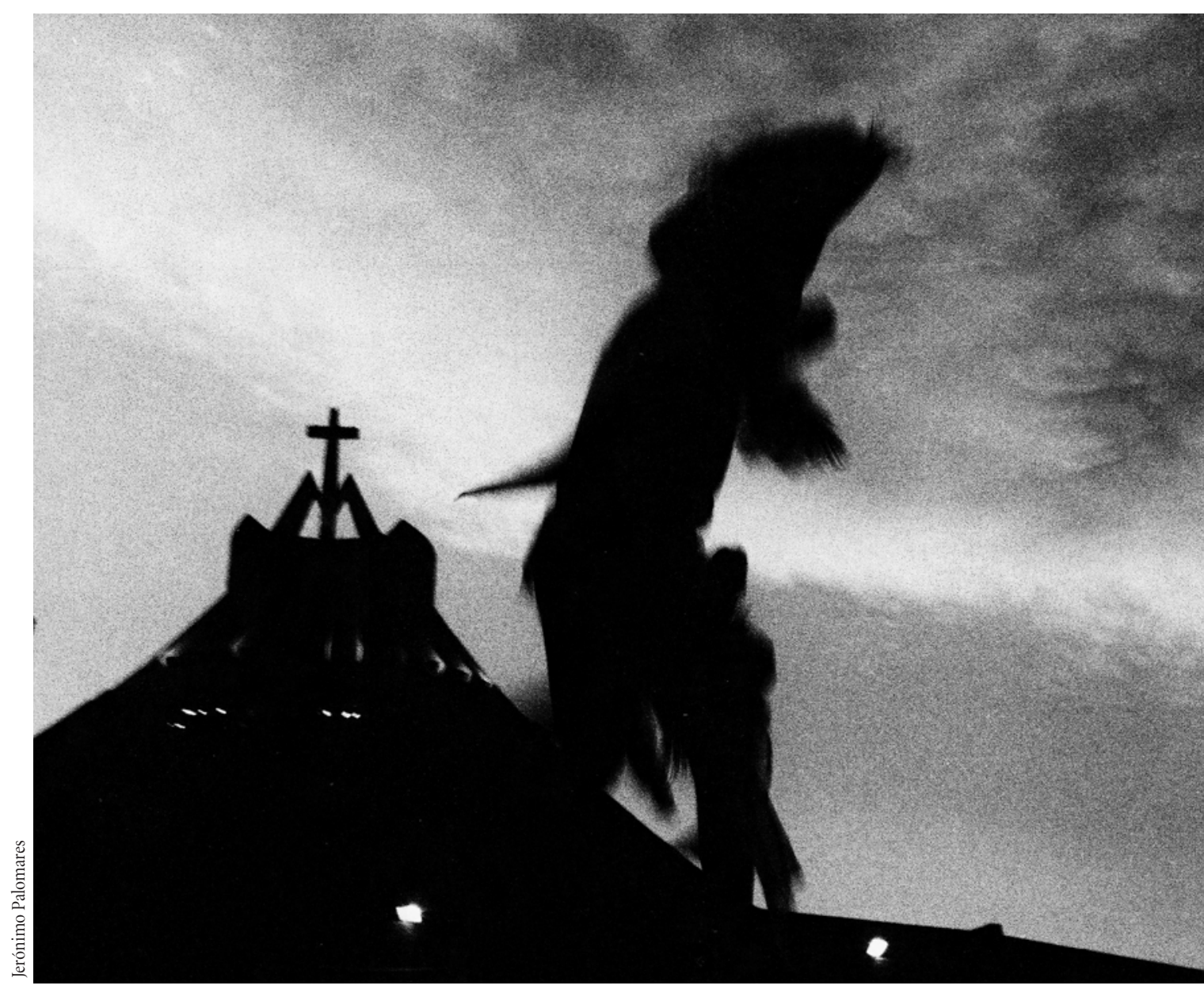

Basílica de Guadalupe, 2004.

informales de carácter religioso que influyen en las decisiones sobre la calidad y el éxito relativo del modelo.

Para probar lo anterior, se elaboró un cuestionario estructurado dirigido a 10\% de los trabajadores de cuatro de los departamentos de esta empresa, compuesto por 68 reactivos con un periodo de aplicación de cuatro meses. La técnica de muestreo para un universo de 800 trabajadores fue aleatoria estratificada con asignación proporcional y un nivel de confianza de 5\%. Asimismo, para conocer las concepciones u opiniones de los gerentes de CIMSA y sus estrategias de dirección y de influencia sobre los mandos medios y los obreros, se recurrió a la realización de entrevistas semiabiertas con el fin de con- trastar sus respuestas con las de los trabajadores acerca de la organización del trabajo, así como sobre las tendencias y estrategias del cambio.

En cuanto a la elección de los departamentos de producción, se consideró a aquellos en los que se encontraban los trabajadores con mayor antigüedad en la empresa, que son los que podrían dar cuenta de los cambios organizacionales y laborales ocurridos en la planta a partir de la implantación del Contrato Colectivo de Trabajo (CTC). Con este criterio se eligió a los departamentos de troquelados y grapas, en los cuales se realiza un trabajo más calificado y donde, curiosamente, sólo laboran hombres. De igual manera, se seleccionó a aquellos departamentos de pro- 
ducción en los que, por un lado, prevalecen formas tradicionales y manuales de trabajo y, por el otro, se trabaja simultáneamente con máquinas automatizadas de producción y nuevas formas organizativas. Éste es el caso de los departamentos de armado de corredera y ensamble final, que son los que efectúan un trabajo de maquila más especializado y están compuestos exclusivamente por mujeres, quienes consideramos podrían tener una concepción particular sobre la forma en que se viven los cambios en la fábrica.

El documento se divide en dos grandes apartados. En el primero se resume parte de la discusión en torno a los cambios y tendencias que registran las nuevas formas de organización del trabajo, así como su relación con una nueva cultura gerencial y laboral en las fábricas manufactureras. Aquí se describe lo que consideramos las características más comunes de un modelo ideal de organización del trabajo, además de los componentes esenciales del modelo organizacional de CIMSA. En el segundo se detallan y analizan los resultados del trabajo de campo, divididos en tres de las vertientes más representativas del modelo: a) lenguaje, discurso y formas de dirección; b) identificación, compromiso y sentido de pertenencia a la empresa, y c) interpretación de los cambios en la organización del trabajo. Al final de estos dos apartados se incluye una sección de conclusiones.

\section{LA ORGANIZACIÓN DELTRABAJO Y LA CULTURA GERENCIAL A LA CONQUISTA DE LA SUBJETIVIDAD LABORAL}

\section{El modelo de organización ideal}

Una definición apropiada para los fines de este estudio va en el sentido de que la organización del trabajo puede ser pensada como un conjunto de prácticas multidimensionales, que si bien se inspira en ideas sistematizadas a partir de una lógica racional-productivista también encarna relaciones de poder cuyas manifestaciones concretas pueden asumir formas de resistencia o de cooperación. Dentro de estas nuevas prácticas organizacionales —representadas a través de corrientes de pensamiento como la escuela es- tadounidense de las nuevas relaciones humanas (NRH) y su estructura jerárquica de necesidades; los nuevos enfoques sociotécnicos y su pretendida organización científica del trabajo; o los también llamados modelos posfordistas, toyotistas, de Lean production o modelo japonés de involucramiento responsable, entre otras denominaciones-ocupa un lugar privilegiado el interés por influir en la subjetividad de los trabajadores como la mejor garantía para la consecución de los objetivos empresariales. Es decir, la organización del trabajo hoy se presenta como la búsqueda planeada de la legitimidad en aras de una mayor productividad, ya que la ganancia ha dejado de estar en función de la manipulación técnica de los recursos, incluida la fuerza de trabajo. Ahora se persigue, con más fuerza que nunca, la conquista de la voluntad (Gatica, 1993: 24).

De manera resumida, podemos decir que las nuevas prácticas organizacionales están contenidas en programas y preceptos filosóficos y motivacionales con los que los trabajadores adquieren un amplio rango de habilidades, a través de la educación, el entrenamiento y la rotación del empleo. Estas prácticas se expresan tanto en normas, valores, creencias, reglas y procedimientos como mediante formas simbólicas o conceptos de trabajo: "mejora continua", "control total de calidad", "trabajo de grupo", "empresa-familia”, etc., con los cuales la gerencia y los trabajadores ordenan su trabajo en un contexto social histórico, con una carga emocional que les da sentido. De hecho, todas estas técnicas de participación, comunicación y motivación — como veremos más adelante— pueden ser interpretadas como formas de subjetivación que coexisten al interior de un campo de posiciones y sirven de guía para reproducir la cultura de la corporación, además de generar sentimientos de pertenencia colectiva ${ }^{3}$.

\footnotetext{
${ }^{3}$ Estos sentimientos de pertenencia colectiva, claro está, no implican que las diferencias de clase social desaparezcan, ya que, como señala Touraine (1984), las clases, como grupos opuestos uno contra otro, en conflicto central para la apropiación de una historicidad hacia la cual ambos se orientan, están definidas por su posición dominante o dependiente en el modo de apropiación de la historicidad y de los modelos culturales de conocimiento y moralidad: "ambos grupos tienen en común las mismas orientaciones culturales: tanto uno como otro creen en el progreso, en la gratificación diferida y en un control represivo de la vida sexual. Sin embargo, al mismo tiempo luchan uno contra otro por el control social de la cultura industrial y por dar diferentes formas sociales a las mismas orientaciones culturales. El me-
} 
Podría señalarse que lo novedoso de las actuales formas de organización del trabajo no está tanto en la utilización de estrategias de convencimiento, indistintamente de los mecanismos utilizados para ello, sino en el énfasis que estas nuevas formas ponen en ese propósito de convencimiento; es decir, en las formas que hacen posible que los trabajadores entreguen voluntariamente su mejor esfuerzo en pos de la calidad y la productividad, sin que ello implique la desaparición de los mecanismos de coerción o represión contra quienes se resistan al sistema de organización. Así, quizá las nuevas propuestas organizacionales son más que eso y su intención abarca, además, el desarrollo de una verdadera "ergonomía del alma" (Gatica, 1993: 11).

Abo (1994) ha señalado que en ausencia de un medio ambiente social y cultural de apoyo como el de Japón, país donde estas tendencias organizativas han tenido mayor desarrollo, la transferencia de este modelo de cultura corporativa a la producción local en países del continente americano ha demandado, de parte de las compañías manufactureras, un esfuerzo concentrado y deliberado por encontrar un campo de interacción aceptable que les permita aplicarlo al máximo. Sin embargo, ésta no ha sido una tarea fácil, ya que los trabajadores, con sus acciones, han mostrado que los cambios técnicos en la organización del trabajo, la flexibilidad asociada a las relaciones laborales y las nuevas "filosofías" de trabajo tienen efectos profundos en su subjetividad, debido a que generan reacciones variadas y diversas, algunas no siempre entendibles ni aceptables para las empresas. Por regla general, la introducción de lo que hoy se conoce como técnicas propias del modelo japonés ha estado acompañada por actitudes ambiguas y contradictorias, que van desde el consenso o el rechazo, hasta otras francamente contemplativas.

La industria manufacturera moderna es un claro ejemplo de cómo los trabajadores se sienten cautivados por los nuevos equipamientos, pero también de la resistencia que muestran a colaborar con las gerencias, con las que comparten interpretaciones distintas acerca de las nuevas

canismo central es el conflicto, a través del cual un campo de historicidad y un conjunto de modelos culturales se transforman en un sistema de relaciones sociales, las cuales son siempre desiguales, pues son relaciones de poder" (Touraine, 1984: 68). formas de organización, dirección y ejecución del trabajo. Por eso cuando diversas empresas, en sus operaciones de producción local, establecen un sistema de dirección y producción inspirado en estas técnicas, se ven obligadas a hacer un enorme esfuerzo para crear las condiciones que permitan impulsar un tipo de cultura corporativa orientado a la apropiación de la subjetividad del trabajador.

A pesar de estos inconvenientes, ya se ha difundido ampliamente la idea generalizada de que nos encontramos ante una generación de jóvenes con nuevas actitudes, creencias, valores y comportamientos en relación con el trabajo, en clara ruptura con los viejos obreros que tienen ideas distintas sobre la calidad de los productos, la tecnología y la productividad (Zurla, 1989-1990). En México, por ejemplo, desde la segunda mitad de la década de 1980, Palomares y Mertens (1987) han catalogado a estas técnicas de involucramiento, comunicación y motivación como propias de un nuevo modelo de cultura corporativa que está generando un patrón conductual mucho más complejo entre los trabajadores, que va más allá de la polarización entre trabajo calificado de programación y trabajo descalificado y rutinario.

Otros autores han considerado estos aspectos como parte de los procesos de "ingeniería cultural" (Reygadas, 1995), a través de los cuales las grandes firmas rediseñan y perfeccionan sus estrategias de organización del trabajo para incidir y moldear los comportamientos laborales en aras de un mayor compromiso con la organización. Otros más los han interpretado como parte de las nuevas "estrategias de racionalización del trabajo" (Neffa, 1982; Pries, 1998; Grzyb, 1989), o bien, como modelos o "procesos de influencia gerencial" para generar consensos y control sobre los trabajadores (De Paula, 1993; Carrillo, 1993; García, 1996). También se ha planteado que no sólo se trata de una atribución que se da la gerencia administrativa para imponer sus intereses corporativos, sino que esto representa además una necesidad lógica de la estructura organizacional que permite poner a prueba un principio de integración social (Abo, 1994) que tiene amplias repercusiones en la subjetividad del trabajador, en sus motivaciones y representaciones simbólicas.

No obstante, fuera de los propósitos del discurso gerencial, cabe la posibilidad de que sólo se trate de una 
nueva fórmula de control social (Coriat, 1992), tendiente a crear obreros amoldados en la cooperación y coordinación de las distintas actividades de trabajo, que sean funcionales para las nuevas tecnologías (sistemas flexibles de fabricación), para los nuevos conceptos de organización de flujo de mercancías (inventarios justo a tiempo), para las exigencias de calidad del mercado y, en general, para el sistema de organización global de la empresa, con lo que se estaría concretando una especie de "racionalidad tecnológica moderna".

Cualquiera que sea el resultado, es un hecho que, hasta hoy, los trabajadores de las plantas manufactureras de México en las que se aplica dicho sistema, como es el caso de CIMSA, están involucrados en un proceso continuo y ascendente de constitución y reconstitución de significados, constantemente valuados y revalorados, reclamados y refutados, lo que permite reconocer una nueva cultura del trabajo. Ésta no es en esencia idéntica a la que opera en las plantas japonesas, pero tampoco es parecida a aquella basada en el puesto de trabajo que prevalecía en las antiguas armadoras mexicanas. En buena medida, esto tiene que ver con las condiciones de adaptación de las empresas y sus trabajadores, en las cuales predominan procesos continuos de experimentación y aprendizaje de la organización. Los problemas que ha engendrado dicho proceso generalmente son de adaptación al entorno y de integración de los recursos humanos internos, lo que al parecer deriva en un modelo de cultura representado por prácticas y concepciones de carácter pragmático, pero a la vez cooperativas y críticas, con características particulares que definen un estilo propio de concebir y desarrollar el trabajo.

\section{EL MODELO DE CIMSA}

Desde 1986, CIMSA ha adoptado una nueva forma de organización del trabajo basada en el Control Total de Calidad (CTC), de la que se desprenden nuevas figuras organizativas, como el Consejo de Calidad y el Consejo Técnico, en las cuales participan los niveles gerenciales de la empresa para diseñar políticas y estrategias en relación con la nueva forma de trabajar: los círculos de calidad
(CC $)^{4}$, y en algunos departamentos de producción los equipos de trabajo. De acuerdo con la gerencia, lo que se busca es la participación de los trabajadores en las propuestas de calidad y en las mejoras del proceso de producción.

Se puede decir que el proceso de producción está sustentado en la filosofía de la empresa, que se supone identificada con el Control Total de Calidad, una calidad que es vista como un concepto integral que abarca la producción, la actualización y la innovación, y que se traduce en "hacer bien las cosas desde el principio". Entre los valores que señala la empresa se plantea que "la gente es lo más importante, sin ella no podríamos cumplir nunca nuestra misión; trabajando en equipo formamos la fuerza y [la] vida de la empresa". No obstante, la Misión de la Dirección es dirigir y administrar los recursos materiales y financieros de la planta para satisfacer los objetivos de servicio y productividad planteados en el Programa de Calidad Total.

Como parte de dichos propósitos se puso en marcha el Programa SOL (Seguridad, Orden y Limpieza), que consiste en que los trabajadores se ocupen de su seguridad, usando el equipo de protección que les brinda la empresa, y se hagan cargo del orden y la limpieza de su área de trabajo. Asimismo, se ha puesto en práctica el sistema de autocontrol de los trabajadores en la producción, lo que, dicho sea de paso, ha implicado el despido de los supervisores o inspectores de calidad de cada departamento. Sin embargo, estos cambios organizativos no contemplan la polivalencia de los trabajadores ni la movilidad horizontal entre puestos o departamentos; es decir, se mantiene el esquema de trabajadores especializados en puestos de trabajo.

Otro aspecto que merece resaltarse se refiere al Día de la Calidad y la Familia, instituido como festejo el 12 de diciembre, fecha en que se celebra a la Virgen de Guadalupe. Ese día se premian los esfuerzos de los trabajadores respecto de la calidad y las ideas que mejoran la produc-

\footnotetext{
${ }^{4}$ Es preciso mencionar que el CC Nueva Imagen de CIMSA fue triunfador del Primer Concurso Nacional de Círculos de Calidad en 1990, por su aportación de dar al cierre la medida exacta, y de igual manera, en 1991, el C C Pioneros ganó el primer lugar en el mismo concurso, por la fabricación de un dispositivo que reduce el ruido en $40 \%$ en su Departamento de Producción.
} 


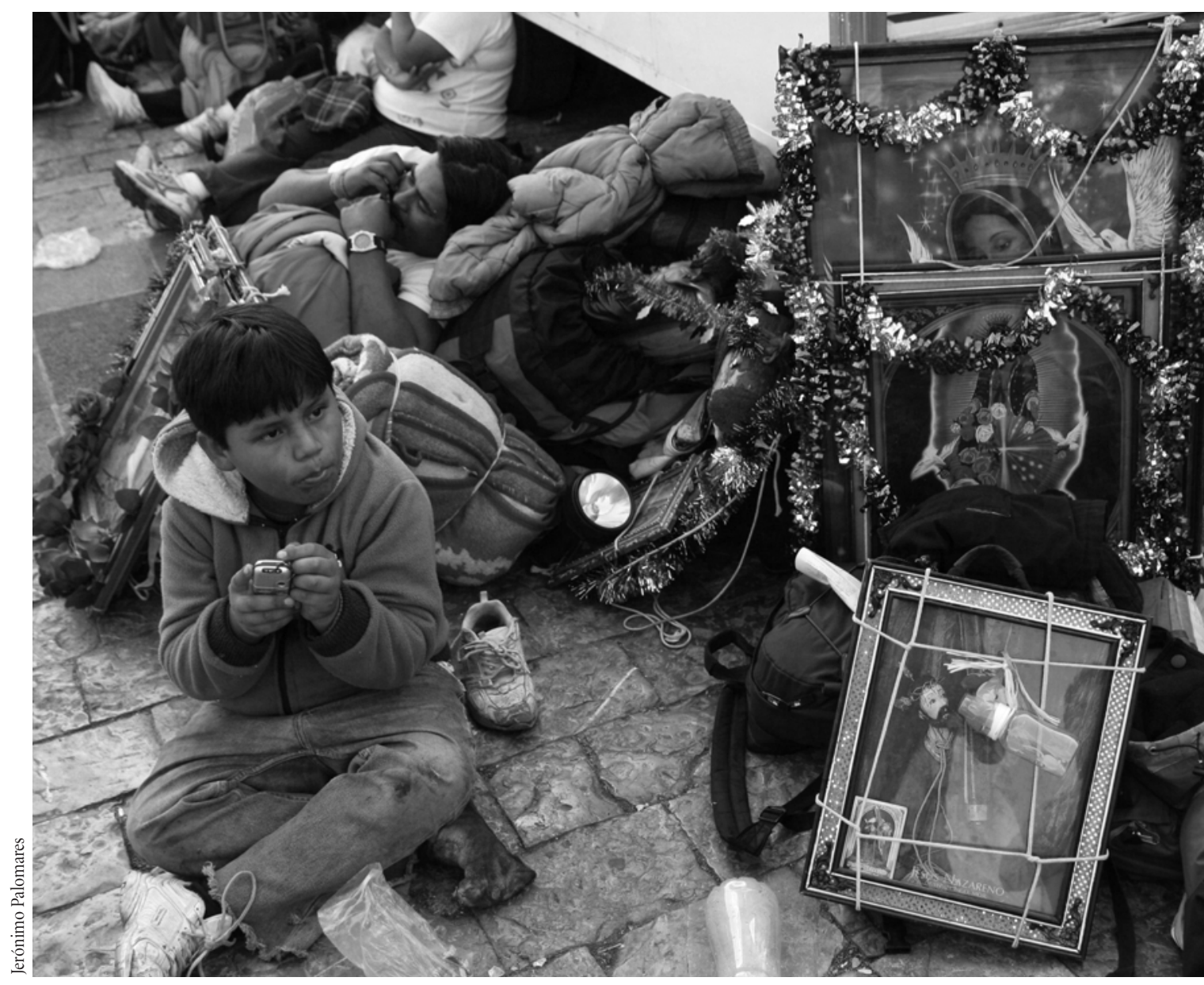

Basílica de Guadalupe, 2008.

tividad. Igualmente, se busca resolver problemas, mejorar productos y relaciones de trabajo. Se reconoce también a los mejores departamentos durante el año, así como a los cC de la empresa.

Desde nuestro punto de vista, todas estas figuras organizativas están relacionadas en CIMSA con el impulso de un principio de involucramiento responsable como factor aglutinador de intereses, alrededor del cual se desarrolla un liderazgo compartido basado en una gestión del personal que pone énfasis en la capacitación y el entrenamiento. A su vez, se las asocia con una comunicación horizontal que se recrea y extiende hacia todos los integrantes de la comunidad de la empresa, entendida ésta como el espacio fundamental en el que se desenvuelve la actividad principal de la gerencia, y en el que ésta invierte no sólo capitales sino valores, conocimientos, tradiciones y demás componentes de una subjetividad y una cultura propias de su papel.

Finalmente, las distintas estrategias orientadas a la búsqueda de la calidad y el involucramiento responsable se mezclan con una cultura gerencial que busca construir una comunidad de intereses, en el sentido de valores compartidos en el ámbito fabril, donde los gerentes ejercen procesos de influencia o de convencimiento sobre sus subordinados y reconfiguran sus concepciones y significados en materia de trabajo gracias a su interacción con 
los sujetos que dirigen. Una cultura que se concibe y que echa mano del discurso de la modernización, la competitividad, la cooperación, la eficiencia y la calidad, y que juega un importante papel en la elección de una alternativa dentro del abanico de posibilidades en materia de organización del trabajo, así como en su forma de relacionarse con los subalternos (véase figura 1). Algunos resultados de este modelo se reflejan al menos en tres aspectos correlacionados: a) lenguaje, discurso y formas de dirección; b) identificación, compromiso y sentido de pertenencia; y c) interpretaciones y divergencias sobre los programas de calidad.

Figura 1. Aspectos básicos del modelo de organización del trabajo en CIMSA

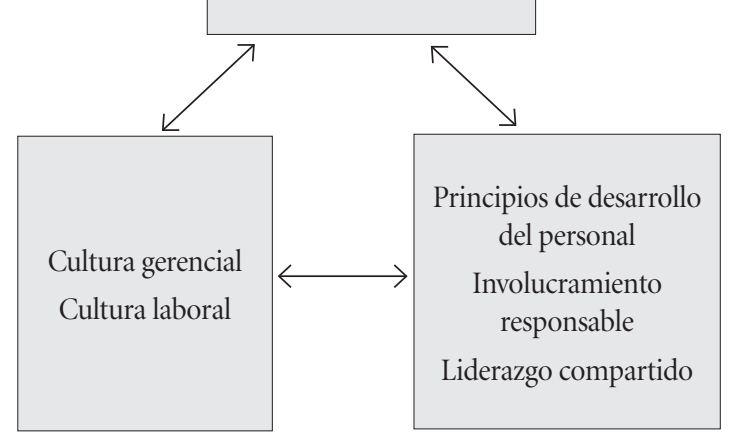

Fuente: Elaboración propia.

\section{RESULTADOS EMPÍRICOS DEL MODELO DE CIMSA}

\section{Lenguaje, discurso y formas de dirección}

La comunicación como aspecto inherente a las formas de dirección es un elemento importante en las estrategias de influencia sobre los trabajadores y los jefes de departamento (JD) o mandos medios (MM) para buscar la im- plantación de nuevos sistemas organizacionales en el trabajo. En este sentido, los gerentes de CIMSA — los jóvenes, los viejos, los profesionistas y los formados por su trabajo en la planta - coinciden en señalar que existe un lenguaje común y elemental para dirigirse a todos los trabajadores. Uno que intenta ser democrático y que se transmite a través del desarrollo del Programa de Calidad Total (PCT). El director general asegura que "anteriormente el lenguaje era muy paternalista, sin embargo, con el PCT siento que el lenguaje que nos une es el de la calidad, porque ahora estamos hablando mucho de la calidad, la productividad, los círculos de calidad [...] éste es un lenguaje que nos ha unido más". En este discurso, el valor de la familia como concepto integrador de los trabajadores juega un papel determinante. Así, resulta significativo el hecho de que la empresa instituyó el 12 de diciembre — día de la Virgen de Guadalupe - como el Día de la Familia y de la Calidad de la Empresa ${ }^{5}$. De hecho, la gerencia reconoce que esta integración es un aspecto fundamental para el desarrollo de CIMSA.

Sin duda alguna, el valor de la calidad ha llegado a ser fundamental en el discurso gerencial, como mecanismo de involucramiento e identificación. No obstante, los JD o MM coincidieron en su apreciación de que el lenguaje universal con el que la empresa se maneja, y que todos entienden, está más orientado al concepto de familia como el valor más importante y la principal estrategia de la gerencia para conseguir la integración de los trabajadores con los principios de la empresa. Adicionalmente, no deja de ser significativo el hecho de que los gerentes, incluyendo a los de nivel medio e inclusive a algunos jefes de

\footnotetext{
${ }^{5}$ A esta fecha se le conoce también como "Aniversario de la Lealtad". Se premia a los departamentos de producción más sobresalientes en calidad y productividad, se otorgan reconocimientos a quienes hayan inventado algún dispositivo o máquina que ayude a la producción, se premian también las ideas de los $\mathrm{CC}$, se reconoce a los trabajadores que cumplen 10, 15, 20, 25, 30 años o más de laborar en la planta y se sortean premios entre los trabajadores y sus familias. La empresa niega que con ello se quiera sacar provecho de los valores festivos de abnegación y respeto que representa esta fecha; sin embargo, resulta difícil pensar que ésta haya sido elegida casualmente, ya que es un día significativo para la tradición católica mexicana, y por lo tanto, para los trabajadores de la planta. Es preciso señalar que todos, o casi todos los departamentos de producción, cuentan con un pequeño altar a la Virgen de Guadalupe.
} 
departamento, se refieran a CIMSA como "nuestra empresa", por lo que se podría decir que entre ellos existe una concepción y una forma común de hablar de la empresa, no obstante que ésta no es una opinión compartida por todos los JD.

Por otra parte, según pudimos observar, la técnica más recurrente de los gerentes para girar instrucciones a sus subordinados es la que ellos definen como "campechaneada" - a veces en forma de sugerencia y en ocasiones como orden-. Para esto, se sabe que dichas órdenes o instrucciones se emiten directamente a los JD, para que ellos a su vez las transmitan a sus trabajadores; es decir, la comunicación entre la gerencia y los operadores o trabajadores de piso no es frecuente; sólo se da en casos especiales, porque se respetan fielmente las jerarquías administrativas.

En general, podría decirse que existe consenso entre todos los JD y supervisores en cuanto a la existencia de un lenguaje común, con aspectos técnicos que sólo ellos pueden entender (no así los trabajadores) y hasta con "malas palabras", que son muy comunes en ambos grupos, y por lo tanto no crean ningún problema. Sin embargo, los supervisores de ensamble final y los JD de armado de corredera, por ejemplo, señalan que la gerencia les ordena en vez de dar instrucciones, y que aunque el lenguaje es claro, existen actitudes arrogantes, despóticas y groseras que les generan incomodidad. En otras palabras, que el trato es más bien distante y autoritario, al grado de que en muchas ocasiones los gerentes ni siquiera se toman la molestia de saludar a los supervisores.

Respecto de la opinión de los trabajadores operarios de línea acerca de la comunicación en la planta, 97\% manifestó que existe muy poca relación entre ellos y los gerentes; es decir, que los últimos no se relacionan o se dirigen a ellos más que en ocasiones extraordinarias, y que con el cambio organizativo no hubo mejoras significativas en las relaciones humanas. En aspectos específicos relativos a la comunicación del director general con los trabajadores, $61.8 \%$ aseguró que el director nunca se dirigía a ellos ${ }^{6}$,

\footnotetext{
${ }^{6}$ Sobre todo las operadoras de ensamble final (77\%), las de armado de corredera (53\%), la mitad de los operadores de troquelados y $42 \%$ de los de grapas.
}

23.7\% afirmó que lo hacía esporádicamente, $10.6 \%$ señaló que a veces lo hacía, y 3.9\% dijo que hablaba con ellos con frecuencia. Considerando lo anterior, tiene coherencia el hecho de que $47 \%$ de los trabajadores de CIMSA declare que desconoce cómo hablan o cuál es el lenguaje de los gerentes de la planta, lo que permite suponer que la comunicación gerencial con los trabajadores es prácticamente escueta o esporádica (véase cuadro 1).

\section{Cuadro 1. El director habla directamente con los trabajadores}

\begin{tabular}{c|c|c|c|c}
\hline $\begin{array}{c}\text { Frecuente- } \\
\text { mente }\end{array}$ & A veces & $\begin{array}{c}\text { Esporádi- } \\
\text { camente }\end{array}$ & Nunca & Total \\
\hline $3.9 \%$ & $10.6 \%$ & $23.7 \%$ & $61.8 \%$ & $100.0 \%$ \\
\hline
\end{tabular}

\section{Identificación, compromiso y sentido de pertenencia}

A la pregunta de si el lenguaje tiene que ver con la conformación de esa comunidad de intereses compartidos en el trabajo, de nuevo, todos los gerentes entrevistados respondieron afirmativamente. En este sentido, el geren-

Sí, mi forma de lenguaje y de dirigirlos ha tenido que ver porque los he dejado ser un poco más libres, más autónomos. Anteriormente se les decía: "haces esto y no pienses", como a los burros de carga que les pones laterales en los ojos para que sólo vean en la dirección que uno quiere; ahora no, ahora el abanico se abrió, ahora se debe buscar desarrollar su creatividad.

En el mismo sentido se expresa el gerente de Calidad Integral, cuando dice que sus trabajadores:

Se sienten muy integrados a la compañía y están muy motivados [porque] he tratado de que hagan las mismas funciones que yo hago; he creado una dinámica dentro del conjunto de personas que estamos trabajando, que cada uno lidere determinado tiempo a todo el equipo, entonces ellos se sienten seguros, se sienten muy integrados a esta familia que de alguna forma somos, y yo creo que es parte esencial para la integración el cómo trasmitirles ese entusiasmo, y realmente ellos son reflejo fiel del jefe.

Por su parte, $78.9 \%$ de los trabajadores de CIMSA manifestó que le gustaba trabajar en la planta, 5.3\% dijo que 
le disgustaba y el 15.8\% restante señaló que le era indiferente (véase cuadro 2). Sin embargo, no deja de ser curioso que sólo $40 \%$ de los que mostraron su gusto por trabajar en CIMSA supiera decir por qué le gustaba su trabajo en la planta; de ese $40 \%$, la mitad dijo que la razón era el gusto por el trabajo que desarrollaban, y el resto se dividió entre los que privilegiaban el ambiente de trabajo $(12 \%)$ y los que siempre habían trabajado allí (7\%), entre otras razones.

\section{Cuadro 2. Gusto por trabajar en la empresa}

\begin{tabular}{c|c|c|c}
\hline Sí & No & Lees indiferente & Total \\
\hline $78.9 \%$ & $5.3 \%$ & $15.8 \%$ & $100.0 \%$ \\
\hline
\end{tabular}

Con la creencia de que una posible forma de identificación o integración de los trabajadores a la empresa podría darse a partir de que la gerencia externara su preocupación por las expectativas o aspiraciones laborales de los trabajadores, se preguntó a los obreros si la empresa mostraba interés en este aspecto, a lo que $57 \%$ de los operadores respondió que no sabía, o que la empresa no se interesaba por estos asuntos. Es decir, que no es posible inferir o concluir que la empresa busque influir sobre los trabajadores a través de estos mecanismos, no obstante que $43 \%$ de los encuestados cree que la gerencia sí se interesa en ellos.

Sin embargo, del total de los que piensan que la empresa se interesa en ellos (43\%), sólo 8\% cree que esto se debe a la preocupación por el desarrollo personal de los trabajadores; $22 \%$ piensa que dicho interés responde a una estrategia para incorporar al proceso de producción los conocimientos prácticos de los trabajadores, y el 12\% restante sostiene que esto se debe al intento de aumentar la calidad y la productividad. En este orden de ideas, resulta curioso que 49\% de los trabajadores de CIMSA haya señalado que creía que la empresa estaba conforme con su trabajo ${ }^{7}$, mientras que otro $49 \%$ dijera que no lo sabía. No obstante, $75 \%$ de los trabajadores de CIMSA confesó sentirse motivado para trabajar en la empresa (véase cuadro 3).

\footnotetext{
${ }^{7}$ Los motivos de esta creencia se dividieron entre los que pensaban que la empresa estaba conforme porque hasta ahora no los habían despedido, porque estaban seguros de su trabajo o porque nunca les habían llamado la atención, entre otras opciones.
}

Cuadro 3. Motivación para trabajar en CIMSA

\begin{tabular}{c|c|c}
\hline Motivado & Desmotivado & Total \\
\hline $75.0 \%$ & $25.0 \%$ & $100.0 \%$ \\
\hline
\end{tabular}

Si ahora nos preguntamos sobre las razones de esta motivación, podremos ver que en la respuesta poco influyen las políticas gerenciales o laborales de la empresa: $40 \%$ de quienes se sienten incentivados para trabajar en la planta atribuyen el hecho a motivos relacionados con su desempeño en el trabajo ${ }^{8}$ - les gusta lo que hacen-o, en menor medida (13\%), a factores económicos, como el bono de productividad. En otras palabras, aquí tampoco podemos encontrar estrategias de convencimiento de parte de la gerencia hacia los trabajadores, ya que sólo $8 \%$ de los encuestados dijo sentirse motivado por la relación con sus superiores, y es preciso aclarar que por superiores se referían a sus jefes de departamento y no a los gerentes.

Por otra parte, $17 \%$ reveló que lo que más le desmotivaba para trabajar en la empresa era el aspecto económico, aunque $75 \%$ argumentó que haría igual su trabajo aun si no se le diera ningún incentivo económico. Esto entra en evidente contradicción con la afirmación de $79 \%$ de los trabajadores que responde que cumple con su trabajo por dinero ${ }^{9}$, o del $28 \%$ que declara hacerlo por los incentivos económicos, lo que podría entenderse como una clara actitud instrumentalista que tiene poco que ver con el sentido de pertenencia y de compromiso con la empresa.

Si conectamos las razones para cumplir con el trabajo con la relación entre trabajadores y JD, podemos obtener datos interesantes. Del 75\% que dijo que haría igual su trabajo aunque no se le dieran incentivos económicos, tenemos que $61.4 \%$ califica su relación con los JD como rígida pero justa, flexible y abierta al diálogo o paternal y humana. Por otro lado, $45 \%$ de quienes advierten que no

\footnotetext{
${ }^{8}$ Así lo señalan 53\% de las operadoras de armado, 8\% de los operadores de troquelados, $33 \%$ de los de grapas y $46 \%$ de las trabajadoras de ensamble final.

${ }^{9}$ Por departamentos, contestaron eso $91 \%$ de las operadoras de ensamble final, $83 \%$ de los operadores de grapas, $67 \%$ de los operadores de troquelados y $59 \%$ de las operadoras de armado de corredera.
} 
harían su trabajo de la misma manera si no se les dieran incentivos económicos plantearon que la relación con los JD es dominante y a veces injusta. Lo anterior se traduce en niveles de identificación con la empresa de 75\%, aunque no demuestra una correlación directa con las estrategias de motivación concebidas por la empresa (véase cuadro 4). Si relacionamos la motivación con la antigüedad en la empresa, observamos que la primera disminuye conforme aumenta la antigüedad, lo que quiere decir que la posible influencia de la gerencia tampoco surte efecto sobre los trabajadores más antiguos de la planta.

\section{Cuadro 4. Identificación con la empresa}

\begin{tabular}{c|c|c|c}
\hline Identificado & Ajeno & No sabe & Total \\
\hline $75.0 \%$ & $25.0 \%$ & - & $100.0 \%$ \\
\hline
\end{tabular}

\section{Interpretaciones y divergencias sobre la calidad}

Aunque se supone que la empresa ha adoptado el CTC e incluso ha desarrollado su propio programa de calidad (РCT), es curioso que la mayoría de los gerentes de la compañía no sepa en qué consiste dicho programa ni sea capaz de definir el CTC. Así, casi todos dicen que el PCT es muy propio de CIMSA y que se ha desarrollado conforme a la idiosincrasia y las políticas de la planta; que se considera la limpieza, la seguridad, la puntualidad, la asistencia, el control estadístico del proceso y el control y manejo de herramientas, entre otros aspectos.

El gerente de Aseguramiento de la Calidad señala que el CTC que se maneja en la empresa puede definirse como el conjunto de acciones y actitudes de todos los miembros de la organización para lograr la calidad de los recursos — productos, instalaciones, máquinas, servicios y, sobre todo, su gente - que se ofrecen para lograr la plena satisfacción del cliente. Agrega que para CIMSA la calidad no se controla sino que se produce, por eso ellos no hablan de control de calidad sino de aseguramiento de la calidad.

Según señala el mismo gerente, el PCT de CIMSA pone énfasis en el diseño del proceso, para que sea el operador directo quien controle su trabajo y su producción; es de- cir, se practica el autocontrol por parte de los trabajadores sobre el proceso de producción, lo que implica que la responsabilidad final del proceso recae directamente en el trabajador ${ }^{10}$.

El JD de Troquelados reconoce que antes cada quien trabajaba por su lado y "ahora no, ahora trabajamos todos de acuerdo, nos piden nuestra opinión [...] ahora es muy bonito, a mí me gusta mucho [...] ha cambiado hasta en la forma de mandarnos, porque antes todo era más rudo". Sin embargo, el JD de Grapas dice:

El PCT está en pañales, lo tenemos desde hace tres o cuatro años pero no ha funcionado, y los primeros que no están convencidos del dichoso programa son nuestros dirigentes, y ellos son los que deben estar más convencidos, pero a ellos lo que les gusta es el show. Si va a venir una visita dicen: "ay, vamos a impresionarla, pongan ahí lo del Programa de Calidad Total y digan que hacen el Programa SOL", y como pertenecen a la Asociación de Empresarios de Iztapalapa, entonces los invitan y se presumen entre ellos, pero se presumen con cosas que no son reales.

Es importante apuntar que las respuestas a la pregunta de qué es el CTC en CIMSA fueron desde "obtener lo que nos pide el cliente en calidad y servicio", hasta "el CTC son todos los conceptos, o sea, tener calidad en todo lo que estamos haciendo", pasando por "es todo lo que tiene que ver con tu forma de ser, desde peinarte, tratar con la gente, etc., pero en CIMSA sólo se busca la calidad sobre el producto y no en el proceso, ni en el trato a los trabajadores, ni en los salarios, porque la calidad en la comunicación entre la gente no se da”. Pese a todo, los JD piensan que la nueva organización del trabajo está orientada al logro de formas de integración del trabajador a la producción, así como a la motivación para mejorar su trabajo. Además, la mayoría también coincide en que con la nueva organización los operadores trabajan tanto como antes; es decir, que no puede decirse que las cargas de trabajo se hayan incrementado.

\footnotetext{
${ }^{10}$ Hasta hace pocos años se tenía una planta de 60 inspectores de calidad que patrullaban el piso de Producción, mientras que recientemente esa planta se redujo a cinco auditores del proceso de producción.
} 
En lo referente a los CC, el JD de Troquelados señala que en un principio se oponía a éstos porque pensaba que eran una pérdida de tiempo, pero ahora los acepta porque se han mejorado las herramientas y la producción. Sin embargo, el resto de los JD cuestionan la eficacia o bondades de los círculos y opinan que:

deberíamos tener otro sistema, no sólo los CC, porque ésos funcionaron al principio - a los japoneses les funcionaron por su educación, pero nosotros somos diferentes-, pero ahora ya no dan resultado. Los integrantes delCC se pelean y compiten entre ellos, los gerentes no pelan a la gente ni a sus propuestas de mejoras y la gente se desencanta, así que necesitamos algo más adecuado a nuestra realidad, porque nosotros no estamos acostumbrados a organizarnos ni a trabajar de esa forma.

Los trabajadores de CIMSA, según cada departamento de producción, entienden de manera distinta los cambios organizativos en la empresa. Los desacuerdos empiezan a dibujarse cuando sólo $27.6 \%$ de la planta señala que estos ajustes tienen como objetivo aumentar la calidad. Al contrario, $65.8 \%$ afirma que el propósito es el incremento de la productividad, la reducción de costos y la fabricación de nuevos productos (véase cuadro 5). Por otro lado, 13\% dice que ha aumentado la eficiencia en la producción, y un porcentaje igual suscribe que el cambio no ha traído ninguna ventaja a la empresa. Por su parte, $21 \%$ observa que los cambios tienen la desventaja de aumentar las cargas de trabajo. Lo que resulta revelador es que $55 \%$ de los trabajadores expresa que estos cambios no los ha beneficiado en nada ${ }^{11}$, y los demás se dispersan entre los que piensan que ha disminuido la carga de trabajo (8\%) o que aprecian los premios a los CC (5\%), entre otras opciones.

Lo que sí queda claro es que, según las encuestas, 57\% de los trabajadores de CIMSA no aceptó los cambios resignada o pasivamente, y sólo 9\% dijo haberlos aceptado sin hacer comentario alguno; es decir, que más de la mitad cuestionó las nuevas reglas y opuso alguna resistencia, aunque al final acató la nueva organización del trabajo. Por otra parte, resulta curioso que sólo 49\% de los entrevistados diga que las formas de trabajar cambiaron con el

\footnotetext{
${ }^{11}$ Sobre todo las operadoras de ensamble (63\%), los operadores de grapas $(58 \%)$, los de troquelados $(50 \%)$ y las operadoras de armado de corredera $(41 \%)$.
}

Cuadro 5. Motivos de cambio organizacional

\begin{tabular}{c|c}
\multicolumn{2}{c}{ La organización del trabajo cambió para reducir costos } \\
\hline$S i ́$ & No \\
\hline $71.1 \%$ & $28.9 \%$ \\
\hline
\end{tabular}

La organización del trabajo cambió para aumentar la calidad

\begin{tabular}{c|c}
\hline Sí & No \\
\hline $27.6 \%$ & $72.4 \%$ \\
\hline \multicolumn{2}{c}{$\begin{array}{c}\text { La organización del trabajo cambió para aumentar } \\
\text { la productividad }\end{array}$} \\
\hline Sí & $34.2 \%$ \\
\hline $65.8 \%$ & No \\
\hline \multicolumn{2}{c}{ La organización del trabajo cambió para poder exportar } \\
\hline $60.5 \%$ & $39.5 \%$ \\
\hline
\end{tabular}

La organización del trabajo cambió para poder ampliar mercados

\begin{tabular}{c|c}
\hline Sí & No \\
\hline $48.7 \%$ & $53.3 \%$ \\
\hline
\end{tabular}

La organización del trabajo cambió para fabricar nuevos productos

\begin{tabular}{c|c}
\hline Sí & No \\
\hline $72.4 \%$ & $27.6 \%$ \\
\hline \multicolumn{2}{c}{ La organización del trabajo cambió para reducir personal } \\
\hline Sí & No \\
\hline $65.8 \%$ & $34.2 \%$ \\
\hline
\end{tabular}

CTC. Para más de la mitad de los trabajadores (51\%), no se ha producido cambio alguno y, por el contrario, aseguran que siguen prevaleciendo serios problemas que impiden instrumentar el Programa tal como se concibió (véase cuadro 6). Así, del 49\% mencionado, únicamente $11 \%$ dice que los cambios se observan en una mayor responsabilidad en el trabajo, y $8 \%$, en el autocontrol de los trabajadores y en una mayor calidad. 
Cuadro 6. Principales problemas para aplicar el PCT

\begin{tabular}{c|c|c|c|c|c|c|c}
\hline \hline $\begin{array}{c}\text { No } \\
\text { respondió }\end{array}$ & $\begin{array}{c}\text { No se respetan } \\
\text { las reglas } \\
\text { del PCT }\end{array}$ & $\begin{array}{c}\text { Los jefes } \\
\text { no saben } \\
\text { cómo hacerlo }\end{array}$ & $\begin{array}{c}\text { Falta de capa- } \\
\text { citación de los } \\
\text { trabajadores }\end{array}$ & $\begin{array}{c}\text { Falta de } \\
\text { planeación de } \\
\text { la gerencia }\end{array}$ & $\begin{array}{c}\text { Las contradic- } \\
\text { ciones de los } \\
\text { gerentes }\end{array}$ & Otros & Total \\
\hline $32.1 \%$ & $6.6 \%$ & $11.3 \%$ & $9.2 \%$ & $27.6 \%$ & $6.6 \%$ & $6.6 \%$ & $100 \%$ \\
\hline
\end{tabular}

\section{CONCLUSIONES}

Los resultados del cruce de encuestas a trabajadores y entrevistas a mandos medios y gerentes reflejan una organización del trabajo relativamente exitosa, aunque contradictoria y defectuosa. El liderazgo gerencial que está en la base de dicha organización no se ha practicado de acuerdo con el modelo o como señalan los teóricos de la calidad. Esto lo constatan tanto jefes de departamento como trabajadores, quienes afirman que el PCT no ha funcionado adecuadamente debido a la falta de planeación y consistencia de la gerencia respecto del manejo de los elementos y características que componen dicho programa.

La primera inconsistencia se presenta con el desarrollo de un PCT o un CTC desvinculados de sus relaciones con el personal encargado de producirlos. Ello tiene que ver con la práctica de un discurso desligado de las expectativas del personal. Los gerentes hablan de relaciones más cercanas con los trabajadores cuando ni siquiera les dirigen la palabra; o promueven CC a los que no atienden como sus integrantes esperan. Asimismo, la empresa pretende una moderna organización del trabajo que abra escenarios a la participación de los operarios en el proceso de producción, pero no hay suficientes espacios para esa participación; se plantea una moderna forma de dirección con estructuras más horizontales y cercanas a los trabajadores, pero estos últimos aseguran que no existe ninguna relación entre ellos y la gerencia; se afirma que se trabaja con nuevas reglas que privilegian la calidad ante todo, pero los obreros se quejan de contradicciones en la aplicación de estas reglas y de la falta de planeación de la gerencia en este sentido.

Lo que se observa en realidad es un control de la calidad discrecional y sui generis, conformado por una combinación de normas, valores, costumbres, tradiciones y formas de razonamiento compartidos de manera parcial por los diferentes actores de la planta. Así, mientras que para la gerencia la calidad es un medio de competencia con otras empresas del mismo ramo y una forma de obtener prestigio ante la comunidad empresarial, para los MM representa incluso una nueva modalidad laboral que puede funcionar si los gerentes se capacitan y se involucran en el proceso de trabajo en vez de dar órdenes desde el escritorio. Por su parte, para los trabajadores el PCT tiene un significado nebuloso y resulta difícil de definir; es una realidad contradictoria que supone para ellos más responsabilidad y pocos beneficios.

Una segunda gran inconsistencia tiene que ver con la dimensión ideológica subjetiva del modelo. La gerencia implementa una serie de normas y valores que combinan elementos de las formas tradicionales de organización burocrática asociadas a los principios del taylorismo fordismo (centralización de decisiones, escasa rotación del personal, formas autoritarias de vigilancia y control, jerarquías marcadas entre gerentes, JD y trabajadores, y poca comunicación entre la cúpula y la base de la estructura organizativa de la empresa) con formas participativas de trabajo en equipo propias de las nuevas formas organizacionales (círculos de calidad, trabajo en equipo, involucramiento responsable, liderazgo compartido, etc.). Prevalece el trabajo con conocimientos especializados en vez de polivalentes; dominan las costumbres y tradiciones rígidas y el respeto por las jerarquías que inhiben la participación de los trabajadores en las decisiones del proceso de producción. La búsqueda de consensos se ve obstaculizada por una forma de control que introduce una concepción y ejecución tramposa del PCT, con modificaciones y aplicaciones "muy a la mexicana" que reconfiguran subjetividades, tradiciones, costumbres y valores empresariales del pasado reciente, y de un presente que "busca la calidad ante todo". La novedad de una organización del trabajo 


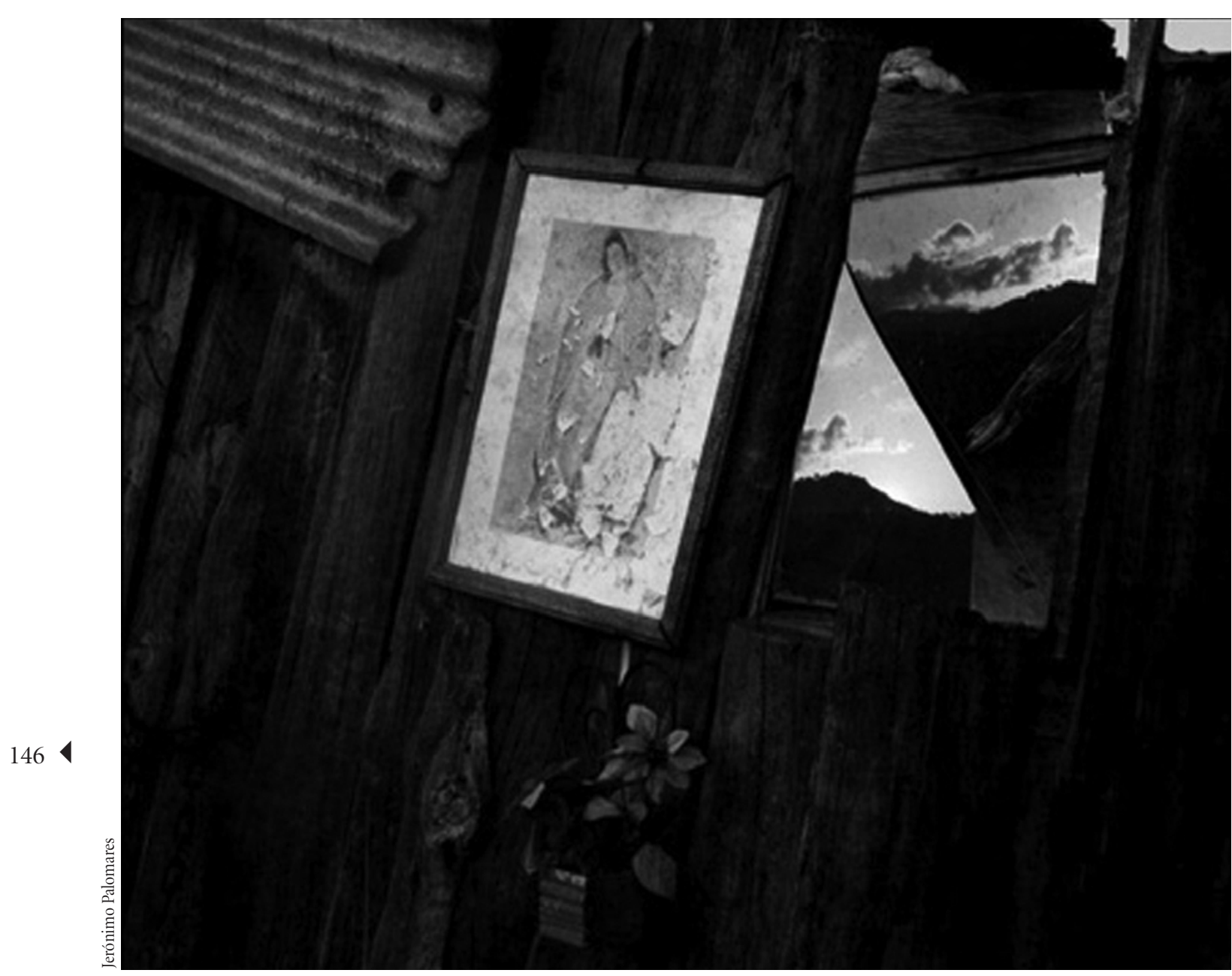

Casa de un ermitaño, 2009.

distinta sería, por lo tanto, aparente, al menos en este sentido, ya que es difícil probar que la empresa ha tenido la facultad de decidir u ordenar la conducta de los trabajadores. Las subjetividades y voluntades de CIMSA no fueron domesticadas, en el sentido de que las nuevas reglas no se aceptaron pasivamente y que a la fecha aún se perciben resistencias a las nuevas formas de trabajar.

Finalmente, un tercer ámbito de contradicciones tiene que ver con las inconsistencias entre el discurso gerencial, que niega el manejo de valores y elementos religiosos al interior de la planta, y las prácticas informales de influencia que lleva a cabo la empresa, en relación con el culto religio- so de los trabajadores. Como es sabido, la religión — como una de las instituciones básicas del capitalismo - introduce valores que tienen una gran influencia en el grado de desarrollo de los pueblos, y en sus actitudes y comportamientos frente al mundo. En el caso de CIMSA, la religión católica constituye un elemento que explica el éxito relativo del nuevo modelo organizacional y del funcionamiento parcial del PCT. La empresa conoce el valor de la religión, en cuanto al fervor guadalupano, y lo ha manejado en estrecha relación con el valor de la calidad; es decir, si la calidad no estaba dentro de la cultura y la escala de valores de los trabajadores, ésta podría ser una forma de incor- 
porarla. Así, CIMSA rescató la cultura popular y el culto a la Virgen de Guadalupe e integró la religión católica como parte de la cultura organizacional de la empresa. De la misma forma introdujo el valor de la familia como elemento fundamental de la ideología y del discurso de la empresa, para convertirse en un modelo ideal de trabajo susceptible de lealtad, respeto y confianza por parte de los trabajadores.

Lo anterior coincide con las observaciones de Ishikawa (1992), quien afirma que la religión tiene mucho que ver con la aplicación del control de calidad, porque mientras las enseñanzas básicas del cristianismo parecen decir que el hombre es malo por naturaleza, en Japón, en cambio, el método Mencius supone lo contrario. En esta idea, Deming (1989), al igual que Ishikawa, aboga por un control de calidad basado en la convicción de la bondad de la gente y en la confianza en los subalternos, porque el control estricto se basa en la convicción de que los hombres son malos por naturaleza y ese sistema no funciona.

Así, los trabajadores de CIMSA, considerados "malos" por naturaleza según la religión que profesan, podrían identificarse como "buenos", en tanto se dicen capaces de trabajar aun sin recibir incentivos económicos; porque se sienten identificados con la empresa aunque la gerencia no los motive, ni tenga comunicación o relación con ellos; porque les gusta trabajar en CIMSA, aunque sus gerentes no sean los mejores — según la evaluación que hicieron de ellos- y no se preocupen por sus expectativas laborales ni convivan nunca con el personal de la planta.

Se podría decir que la "familia ideal guadalupana" de CIMSA, con todo y su PCT, está sustentada en bases muy endebles, en cuanto a una "nueva" organización del trabajo que muestra serias contradicciones en su diseño y ejecución; en cuanto a un proceso de influencia gerencial o de liderazgo interrumpido por los mandos medios, que mediante la reconfiguración de las instrucciones que vienen de la gerencia — en relación con el manejo de lo ideológico subjetivo y el desarrollo de prácticas de influencia informales y de relaciones de dirección más estrechas con los trabajadores- parecen tener más poder de convencimiento y consenso ante los obreros que la figura representativa de lo racional legal que maneja la gerencia. No es pues la gerencia la que tiene el liderazgo total sobre los trabajadores, pues los MM son quienes pueden garantizar el cumplimiento del trabajo de cada departamento, independientemente de que se respeten o no las nuevas disposiciones del PCT.

En resumen, el PCT es más bien un programa tecnocéntrico, autoritario y discrecional maquillado con ingredientes cosméticos de un modelo antropocéntrico que choca con un discurso gerencial contradictorio, el cual no logra resolver los problemas de adaptación y de integración de los recursos humanos. En consecuencia, esa "ergonomía del alma" que debiera experimentarse entre los distintos actores de la empresa se encuentra estancada por falta de un liderazgo lo suficientemente capaz de conciliar las subjetividades y las distintas sociedades culturales que se enfrentan en el seno de la familia de Cierres Ideal de México, S.A.

\section{Bibliografía}

Abo, Tetsu (ed.), 1994, Hybrid Factory: The Japanese Production System in the United States, Oxford University Press, Nueva York.

Coriat, Benjamin, 1992, Pensar al revés: trabajo y organización en la empresa japonesa, Siglo XXI, México.

Carrillo, Jorge, 1993, La Ford en México: reestructuración industrial y cambio en las relaciones sociales, tesis doctoral, El Colegio de México, México.

Deming W., Edwards, 1989, Calidad, productividad y competitividad; la salida de la crisis, Ediciones Díaz de Santos, S. A., Madrid.

De Paula Leite, Marcia, 1993, "Innovación tecnológica y subjetividad obrera”, Sociología del Trabajo, núm. 19, pp. 3-26.

Gatica Lara, Ignacio, 1993, Organización del trabajo fabrily subjetividad de los trabajadores, avance de tesis, mimeo.

Grzyb, Gerard J., 1989, "Descolectivización y recolectivización en los lugares de trabajo: los efectos de la tecnología sobre los grupos de trabajo informales y la cultura del trabajo", en Juan José Castillo (comp.), Las nuevas formas de organización del trabajo, viejos retos de nuestro tiempo, Colección Informes, núm. 3, Ministerio de Trabajo y Seguridad Social, España.

García Figueroa, Gabriela, 1996, Cultura e influencia gerencial en la empresa, un estudio de caso, tesis de maestría en Sociología del Trabajo, Universidad Autónoma MetropolitanaIztapalapa, México.

Ishikawa, Kaoru, 1992, ¿Qué es el control total de calidad? La modalidad japonesa, Grupo Editorial Norma, Colombia. 
Neffa, Julio César, 1982, Proceso de trabajo, división del trabajo y nuevas formas de organización del trabajo, Colección Cuadernos in et, núm. 20, Instituto Nacional de Estudios del Trabajo y Secretaría del Trabajo y Previsión Social, México.

Pries, Ludger, 1998, "Relaciones industriales en la industria automotriz", en R. Dombois y L. Pries (comps.), Las relaciones industriales en el proceso de transformación en América Latina, el caso de México. Proyecto comparativo internacional: transformación económica y trabajo en América Latina, Universidad de Bremen, Universidad de Colombia, Centro Brasileiro de Análise e Planejamento, Universidad Autónoma Metropolitana-Iztapalapa, El Colegio de Puebla, México.
Palomares, Laura A. y Leonard Mertens, 1987, "El surgimiento de un nuevo tipo de trabajador en la industria de alta tecnología: el caso de la electrónica”, Análisis Económico, vol. VI, núm. 10, México, pp. 31-53.

Reygadas, Luis, 1995, “Chinos, indios y gabachos: la intersección de culturas nacionales del trabajo en maquiladoras de México y Guatemala”, ponencia, Xx Congreso de la Asociación Latinoamericana de Sociología, México.

Touraine, Alain, 1984, El regreso del actor, Eudeba, Buenos Aires.

Zurla, Paolo, 1989-1990, "Calidad y cultura del trabajo en los años ochenta”, Sociología del Trabajo, núm. 8, pp. 109-134. 